\title{
TRANSVERSALITY IN $G$-MANIFOLDS
}

\author{
BY
}

M. J. FIELD

\begin{abstract}
A definition of transversality is given for the category of $G$-manifolds ( $G$, a compact Lie group). Transversality density and isotopy theorems are shown to hold for this definition. An example is given to show that we cannot require differential stability of intersections.
\end{abstract}

1. Introduction. Let $G$ be a compact Lie group. This paper is devoted to an extension of transversality theory to $G$-manifolds. The theorems we prove were motivated by earlier work on equivariant dynamical systems (see [3], [4]) and in [6] we apply the results of this paper to give a Kupka-Smale density theorem for equivariant dynamical systems. The reader is also referred to recent work of Poenaru on the stability theory of equivariant maps [13], [14] and to the work of Petrie [12]. We feel that the main significance of the results presented here probably lies in the way that we construct "localisations" of geometric problems associated to a compact Lie group action and the use of nonlinear techniques where representation theory apparently fails to give adequate information.

For technical reasons we defer our definition of $G$-transversality until $\$ 5$. We may, however, summarize our main result by

THEOREM. Suppose that $M$ and $N$ are $G$-manifolds and $W$ is a closed $G$-invariant submanifold of $N$. Let $C_{G}^{\infty}(M, N ; r)$ denote the space of $C^{\infty}$ equivariant maps of $M$ into $N$ with the Whitney $C^{r}$-topology. There exists $d$, $1 \leqslant d \leqslant \infty$, such that if $r \geqslant d$ we may find an open dense subset $Z \subset$ $C_{G}^{\infty}(M, N ; r)$ with the following property:

Given $f \in Z$, there exists an open neighbourhood $V$ of $f$ in $Z$ such that $g^{-1}(W)$ is continuously equivariantly isotopic to $f^{-1}(W)$ in $M$ for all $g \in V$.

Moreover, if $f \in Z$, the intersection $f^{-1}(W)$ is given locally by equisingular families of algebraic varieties.

In general, we cannot require that intersections are differentiably stable.

We now briefly describe the contents of this paper by section. In $\$ 2$, we

Received by the editors September 4, 1975 and, in revised form, December 30, 1975 and February 24, 1976.

AMS (MOS) subject classifications (1970). Primary 57D35, 57E15.

Key words and phrases. Compact Lie group, equivariant differential topology, equisingularity, stratification, Malgrange division theorem, transversality. 
give some examples of $Z_{2}$-transversality showing that even in the simplest cases we have to allow for the intersections being singular. In \$3 we develop the theory of equivariant maps on linear $G$-spaces. Using the Malgrange division theorem we show that if $V$ and $W$ are linear $G$-spaces we have a naturally defined finite dimensional vector space $M$ and $\operatorname{map} \gamma: C_{G}^{\infty}(V, W)$ $\rightarrow M$. The map $\gamma$ is used in $\S 5$ to give our local definition of $G$-transversality. $\$ 4$ is mainly an application of stratification theory to problems in equisingularity. If $X$ is a semianalytic subset of $R^{m} \times R^{k}$, we show that we may construct a sequence $A_{0} \supseteq \cdots \supseteq A_{k}$ of semianalytic subsets of $R^{k}$ (a "fundamental equisingularity sequence") which provides a hierarchy of local equisingularity types for $X$ along $\{0\} \times R^{k}$. If $X$ is an algebraic variety, the sets $A_{j}$ are all semialgebraic. We also state various isotopy lemmas, all of which follow straightforwardly from our definitions or Thom's first isotopy lemma. In $\$ 5$ we give our local definition of $G$-transversality. This depends on the map $\gamma$ referred to above and is expressed in terms of transversality conditions relative to a fundamental equisingularity sequence. In $\$ 6$ we define $G$-transversality on manifolds and prove an equivariant isotopy theorem for $G$-transversal maps. We also give an example showing that we cannot generally expect differential stability of intersections. In $\$ 7$ we give the easy proof that the set of equivariant maps $G$-transversal to a $G$-invariant submanifold is dense in the Whitney $C^{\infty}$ topology.

It is assumed throughout the paper that the reader is familiar with basic transversality theory and the definition of the Whitney $C^{r}$ topology. References for basic transversality theory are provided by [1], [7].

I would like to thank the referee for many helpful and constructive criticisms of this paper. These have resulted in a substantial improvement and simplification of much of the paper, especially $\S \S 3$ and 5.

After circulating a preprint of the first part of this paper, I learnt from $E$. Bierstone that he had also developed a theory of $G$-transversality (see [2a]). His definition of $G$-transversality appears to be slightly different from the version formulated here (see the remarks at the end of $\S 6$ ).

2. Examples of $Z_{2}$-transversality. In this section we present some simple examples of equivariant transversality for the special case of a $Z_{2}$-action. All maps will be assumed $C^{\infty}$ and we shall emphasise transversality problems in a neighbourhood of the fixed set of the $Z_{2}$-action.

Suppose that $Z_{2}$ acts on $R$ as multiplication by \pm 1 . A map $f: R \rightarrow R$ is $Z_{2}$-invariant if, for all $x \in R, f(-x)=-f(x)$. In particular, we always have $f(0)=0 . f$ will be transversal to $0 \in R$ at 0 if and only if $d f / d x(0) \neq 0$. If $f$ is not transversal to 0 at 0 , we may find a perturbation $f^{\prime}$ of $f$ which is transversal to 0 at 0 (for example, we may assume $\left(f^{\prime}-f\right)(x)=\varepsilon x$, for $x$ small and $\varepsilon \neq 0$ ). Therefore, no obstructions to transversality appear in this 
case. We next consider $Z_{2}$-invariant maps $f: R^{2} \rightarrow R$, where we take coordinates $(t, x)$ on $R^{2}$ and $(x)$ on $R$ and $Z_{2}$-actions $(t, x) \mapsto(t,-x)$ and $(x) \mapsto(-x)$ on $R^{2}$ and $R$ respectively. Since $f$ is $Z_{2}$-invariant, $f(t, 0)=0$ for all $t \in R$ and so $f$ maps the $t$-axis of $R^{2}$ to the origin of $R$. We consider the transversality of $f$ to $0 \in R$ along points of the $t$-axis of $R^{2} . f$ is transversal to 0 at $(t, 0)$ if and only if one of $D_{1} f, D_{2} f$ does not vanish at $(t, 0)$. The Malgrange division theorem implies that $f$ is divisible by $x$ and that there exists a $C^{\infty}$ map $g: R^{2} \rightarrow R$ such that

$$
f(t, x)=x g(t, x) \text {. }
$$

(See $\S 3$ for justification of this statement.) It follows that $D_{1} f$ vanishes identically on the $t$-axis and so $f$ is transversal to 0 at $(t, 0)$ if and only if $g(t, 0) \neq 0$. If we take $f(t, x)=x t, g(t, 0)=t$ and we see immediately that it is not possible to find a $Z_{2}$-invariant map close to $f$ which is transversal to 0 near $(0,0)$. Hence, even in the simplest cases, we stably have points of nontransversal intersection. A naive way round this problem is to require transversality within sets of points of the same $Z_{2}$-orbit type. This type of transversality was previously defined in [4] and referred to as "weak $G$-transversality". Whilst it is easy to prove a density theorem for weakly $G$-transversal maps, there is no hope of proving any type of stability theorem as we now show. We define $f: R^{2} \rightarrow R$ by $f(t, x)=x^{5} . f$ is certainly $Z_{2}$-invariant and is weakly $Z_{2}$-transversal to 0 since the intersection of $f$ with 0 is equal to 0 -the fixed point set of the $Z_{2}$-action. Choosing $a$ small and positive, we perturb $f$ to $f^{\prime}(t, x)=x^{5}-2 a x^{3}+a^{2} x$. $f^{\prime}$ is nontransversal to 0 along the lines $x= \pm \sqrt{a}$.

A much more satisfactory approach towards obtaining a transversality theory for $Z_{2}$-invariant maps $f: R^{2} \rightarrow R$ is to require that $D_{2} f=g: R \rightarrow R$; $t \mapsto g(t, 0)$, is transversal to $0 \in R$. This implies that the points of nontransversal intersection on the $t$-axis are isolated and stable under perturbations of $f$. Suppose that $g(0,0)=0$ and that this additional transversality condition holds. That is, we assume $D_{1} g(0,0)=D_{12} f(0,0) \neq 0$. We investigate the intersection of $f$ with 0 near $(0,0)$. Clearly,

$$
f^{-1}(0)=\{t \text {-axis }\} \cup\{(t, x): g(t, x)=0\} .
$$

The condition $D_{1} g(0,0) \neq 0$, together with the implicit function theorem, implies that there exists a $C^{\infty}$ map $x \mapsto u(x)$, defined on some neighbourhood of zero, such that all solutions of $g=0$ near zero are given by the curve $x \mapsto(u(x), x) \subset R^{2}$. Hence the nontrivial part of the intersection is given by the graph of a $C^{\infty}$ function. Moreover, if $f^{\prime}$ is $C^{r}$ close to $f$, the corresponding $g^{\prime}$ is $C^{r-1}$ close to $g$ and so, by a parametrized version of the implicit function theorem, $u^{\prime}$ is $C^{r-1}$ close to $u$. Hence we have local stability of intersections. Notice, though, that $f^{-1}(0)$ has a singularity at $(0,0)$ and so is 
not a submanifold of $R^{2}$. Finally, the transversality condition on $g$ implies that, near zero, $f$ is transversal to 0 (usual definition) off the $t$-axis (this also follows from the local stability of intersection). Hence our transversality condition on $D_{2} f$ on the fixed point set of the $Z_{2}$-action not only implies stability of intersection near the fixed point set but also that intersections are transversal off, but close to, the fixed point set. Given these properties of $f$, it is now an easy matter to perturb $f$ outside of a neighbourhood of the $t$-axis so as to require that it is transversal to 0 on the whole of the complement on the $t$-axis. We shall not give details as these facts follow from the more general theory that we present in later sections.

3. Linear $G$-spaces. Let $G$ be a compact, not necessarily connected, Lie group and suppose that $V$ and $W$ are finite dimensional linear $G$-spaces (that is, $V$ and $W$ are associated to representations of $G$ ). We shall assume throughout this section that the $G$-spaces $V$ and $W$ remain fixed. All our constructions and definitions will only depend on the isomorphism class of the representations of $G$ on $V$ and $W$. We let $P_{G}(V, W)$ and $C_{G}^{\infty}(V, W)$ respectively denote the sets of equivariant polynomials and $C^{\infty}$ maps from $V$ to $W$. We also let $P_{G}(V)$ and $C_{G}^{\infty}(V)$ denote the rings of $G$-invariant real valued polynomials and $C^{\infty}$ functions on $V$ respectively. If $G$ acts trivially, we drop the subscript $G$ and write, for example, $C^{\infty}(V, W)$ for the set of $C^{\infty}$ maps from $V$ to $W$.

$P_{G}(V, W)$ has the structure of a $P_{G}(V)$-module and, using the standard properties of Haar measure together with the Hilbert basis theorem, it follows easily that $P_{G}(V)$ is a Noetherian ring and that $P_{G}(V, W)$ is a finite $P_{G}(V)$-module. Rather less trivially we have

LEMMA 3.1 (SEE ALSO [14]). Let $\left\{G_{1}, \ldots, G_{s}\right\}$ be a set of generators for the $P_{G}(V)$-module $P_{G}(V, W)$. Then $\left\{G_{1}, \ldots, G_{s}\right\}$ is a set of generators for the $C_{G}^{\infty}(V)$-module $C_{G}^{\infty}(V, W)$.

Proof. Relative to the topology of uniform convergence of functions and all their derivatives on compact subsets of $V, P(V, W)$ is a dense subset of $C^{\infty}(V, W)$. Averaging over $G$ it follows that $P_{G}(V, W)$ is a dense subset of $C_{G}^{\infty}(V, W)$. It is therefore sufficient to show that the submodule of $C_{G}^{\infty}(V, W)$ generated by $\left\{G_{1}, \ldots, G_{s}\right\}$ is closed. But, by the Malgrange division theorem (see [17, Chapter VI, Corollary 1.5]) $\left\{G_{1}, \ldots, G_{s}\right\}$ generates a closed submodule of $C^{\infty}(V, W)$ over $C^{\infty}(V)$. The result follows by averaging over $G$.

REMARK. We may clearly require that generators of $P_{G}(V, W)$ are homogeneous and we shall assume this for the rest of the section.

Definition 3.2. Let $\left\{F_{1}, \ldots, F_{k}\right\}$ be a (homogeneous) set of generators for $P_{G}(V, W)$. We say that $\left\{F_{1}, \ldots, F_{k}\right\}$ is a minimal set of generators for 
$P_{G}(V, W)$ (an "MSG") if no proper subset of $\left\{F_{1}, \ldots, F_{k}\right\}$ generates $P_{G}(V, W)$ over $P_{G}(V)$.

The following elementary lemma, pointed out to us by the referee, results in a considerable simplification of our earlier presentation.

Lemma 3.3. Let $\left\{F_{1}, \ldots, F_{k}\right\}$ be an $M S G$ for $P_{G}(V, W)$. Then any relation of the form

$$
\sum_{j=1}^{k} p_{j} F_{j}=0, \quad p_{j} \in P_{G}(V),
$$

implies that $p_{j}(0)=0,1 \leqslant j \leqslant k$.

Proof. If $p \in P_{G}(V)$, we let $p^{(l)}$ denote the homogeneous part of $p$ of degree $l$. Let degree $F_{j}=d_{j}$. For $1 \leqslant i \leqslant k$, we have

$$
-p_{i} F_{i}=\sum_{j \neq i} p_{j} F_{j} \text {. }
$$

Taking homogeneous parts of degree $d_{i}$, we see immediately that

$$
-p_{i}(0) F_{i}=\sum_{j \neq i} p_{j}^{\left(d_{i}-d_{j}\right)} F_{j}
$$

Hence $p_{i}(0)=0$ by the minimality of $\left\{F_{1}, \ldots, F_{k}\right\}$.

Let $\Re^{\infty}=\left\{h \in C_{G}^{\infty}(V): h(0)=0\right\}$ and $\Re=P_{G}(V) \cap \Re^{\infty}$.

LEMMA 3.4. With the above notation we have

(1) The inclusion $P_{G}(V, W) \rightarrow C_{G}^{\infty}(V, W)$ induces an isomorphism

$$
\begin{aligned}
& C_{G}^{\infty}(V, W) / \Re^{\infty} C_{G}^{\infty}(V, W) \\
& \approx P_{G}(V, W) / \Re P_{G}(V, W) \text { (as vector spaces). }
\end{aligned}
$$

(2) An $M S G$ for $P_{G}(V, W)$ maps to a vector space basis of $P_{G}(V, W) / \Re P_{G}(V, W)$.

Proof. (1) follows from Lemma 3.1, (2) from Lemma 3.3.

REMARKS. It follows from (2) of Lemma 3.4 that the number of elements in an MSG depends only on the $G$-spaces $V$ and $W$. Moreover, it is also easily seen that degree $\left.F_{j}: 1 \leqslant j \leqslant k\right\}$ is independent of the choice of MSG for $P_{G}(V, W)$.

Set $P_{G}(V, W) / \Re P_{G}(V, W)=M$ and let $\gamma: C_{G}^{\infty}(V, W) \rightarrow M$ denote the projection map given by Lemma 3.4. Fix an MSG $\left\{F_{1}, \ldots, F_{k}\right\}$ for $P_{G}(V, W)$ and take the corresponding vector space basis for $M$. In this way we determine a coordinate system on $M$ and isomorphism $M \cong R^{k}$. Given $f \in C_{G}^{\infty}(V, W)$, there exist $p_{j} \in C_{G}^{\infty}(V)$ such that

$$
f(x)=\sum_{j=1}^{k} p_{j}(x) F_{j}(x)
$$


and $\gamma(f)=\left(p_{1}(0), \ldots, p_{k}(0)\right) \in R^{k}$. A change of MSG to $\left\{F_{1}^{\prime}, \ldots, F_{k}^{\prime}\right\}$ induces a linear isomorphism $A: R^{k} \rightarrow R^{k}$ such that $\gamma^{\prime}(f)=A(\gamma(f))$, where $\gamma^{\prime}: C_{G}^{\infty}(V, W) \rightarrow R^{k}$ is the map induced from $\left\{F_{1}^{\prime}, \ldots, F_{k}^{\prime}\right\}$.

Let $d=\max \left\{\right.$ degree $F_{j}$ \}. By the remarks above, $d$ is independent of our choice of MSG. If $f \in C_{G}^{\infty}(V, W)$ vanishes at 0 to order $>d$, then $f \in$ $\mathfrak{N}^{\infty} C_{G}^{\infty}(V, W)$ and so, letting $P_{G}^{d}(V, W)$ denote the space of equivariant polynomials of degree $\leqslant d, \gamma$ factors as follows

$$
C_{G}^{\infty}(V, W) \stackrel{T}{\rightarrow} P_{G}^{d}(V, W) \stackrel{\dot{\gamma}}{\rightarrow} M
$$

where $T(f)=$ Taylor series of $f$ at 0 to order $d$ and $\tilde{\gamma}=\gamma \mid P_{G}^{d}(V, W)$. Hence $\gamma: C_{G}^{\infty}(V, W) \rightarrow M$ is continuous if we give $C_{G}^{\infty}(V, W)$ the Whitney $C^{r}$ topology, $r \geqslant d$ (see [7] for definition of the Whitney topology).

For future reference we now wish to state parametrized versions of some of the above results. The proofs of these results are all straightforward and we shall omit them.

LEMMA 3.5. Let $U$ be a trivial linear $G$-space and $\left\{F_{1}, \ldots, F_{k}\right\}$ be an $M S G$ for $P_{G}(V, W)$. Then $\left\{F_{1}, \ldots, F_{k}\right\}$ is an $M S G$ for $P_{G}(V \times U, W)$.

REMARK. If $W^{\prime}$ is a linear $G$-space and $\left\{G_{1}, \ldots, G_{s}\right\}$ is an MSG for $P_{G}\left(V, W^{\prime}\right)$, then $\left\{F_{1}, \ldots, F_{k}, G_{1}, \ldots, G_{s}\right\}$ is an MSG for $P_{G}\left(V, W \times W^{\prime}\right)$.

From now on we suppose that $U$ is a fixed trivial linear $G$-space. Let $f \in C_{G}^{\infty}(V \times U, W)$. Given $t \in U$, we define $f_{t} \in C_{G}^{\infty}(V, W)$ by

$$
f_{t}(x)=f(x, t), \quad x \in V \text {. }
$$

We now define

$$
\gamma: C_{G}^{\infty}(V \times U, W) \rightarrow C^{\infty}(U, M)
$$

by $\gamma(f)(t)=\gamma\left(f_{t}\right), t \in U$, where $\gamma\left(f_{t}\right)$ is as defined previously.

LEMMA 3.6. The map $\gamma: C_{G}^{\infty}(V \times U, W) \rightarrow C^{\infty}(U, M)$ is continuous if we give $C^{\infty}(U, M)$ the Whitney $C^{r}$-topology and $C_{G}^{\infty}(V \times U, W)$ the Whitney $C^{s+d}$-topology, where $s \geqslant r$.

REMARK. To avoid technical difficulties we have restricted attention in this section to $C^{\infty}$ equivariant maps. In fact, by a theorem of Wasserman [19], any $C^{r}$ equivariant map may be $C^{r}$ approximated by a $C^{\infty}$ equivariant map, $r \geqslant 1$. For a $C^{r}$ theory, $r<\infty$, it is sufficient to have a $C^{r}$ version of Lemma 3.1. Unfortunately, as the referee pointed out to us, the obvious $C^{r}$ version of Lemma 3.1 is false as $C_{G}^{r}(V, W)$ need not be finitely generated as a $C_{G}^{r}(V)$ module.

For the remainder of this section we wish to discuss some semigroups of transformations of $M$ naturally associated to the $G$-spaces $V$ and $W$. We use the subscript " $o$ " to denote origin preserving maps. We shall define actions of 
$C_{G}^{\infty}(V, V)_{o}$ and $C_{G}^{\infty}(W, W)_{o}$ on $M$, acting on the right and left respectively.

Let $t \in M$ and suppose that $\gamma(f)=t$, where $f \in C_{G}^{\infty}(V, W)$. If $g \in$ $C_{G}^{\infty}(V, V)_{o}$ and $h \in C_{G}^{\infty}(W, W)_{o}$, we define

$$
(t) \Omega(g)=\gamma(f g), \quad \Pi(h)(t)=\gamma(h f) .
$$

It is easily verified that $\Omega(g)$ and $\Pi(h)$ are well-defined $C^{\infty}$ origin preserving maps of $M$. Furthermore,

$$
\begin{array}{ll}
\Omega\left(g_{1}\right) \Omega\left(g_{2}\right)=\Omega\left(g_{1} g_{2}\right), & g_{1}, g_{2} \in C_{G}^{\infty}(V, V)_{o}, \\
\Pi\left(h_{1}\right) \Pi\left(h_{2}\right)=\Pi\left(h_{1} h_{2}\right), & h_{1}, h_{2} \in C_{G}^{\infty}(W, W)_{o}
\end{array}
$$

and so $\Omega: C_{G}^{\infty}(V, V)_{o} \rightarrow C^{\infty}(M, M)_{o}$ and $\Pi: C_{G}^{\infty}(W, W)_{o} \rightarrow C^{\infty}(M, M)_{o}$ are semigroup homomorphisms.

REMARKs. (1) $\Omega(g)$ depends only on the germ of $g$ at zero, $g \in C_{G}^{\infty}(V, V)_{o}$.

(2) The definition of $\Pi$ given above also defines $\Pi$ as a map from $C_{G}^{\infty}(W, W)$ to $C^{\infty}(M, M)$. However, in the sequel we always suppose that $\Pi$ is defined on $C_{G}^{\infty}(W, W)_{o}$.

Let Iso $(M) \subset L(M, M)$ denote the group of invertible linear transformations of $M$ and $P(M, M)$ be the set of polynomial maps from $M$ to $M$.

We omit the easy proof of the next lemma.

LEMmA 3.7. $\Omega\left(C_{G}^{\infty}(V, V)_{o}\right) \subset L(M, M)$ and $\Omega$ restricts to a group homomorphism

$$
\Omega: \operatorname{Diff}_{G}(V)_{o} \rightarrow \operatorname{Iso}(M) .
$$

(Diff $_{G}(V)_{o}$ denotes the group of origin preserving $C^{\infty}$ equivariant diffeomorphisms of $V$.)

$W$ decomposes uniquely as a direct sum of linear $G$-spaces $W_{0} \oplus W_{1}$, where $G$ acts trivially on $W_{1}$ and does not act trivially on any proper subspace of $W_{0}$ (in case $W_{1}=W$, we take $W_{0}=\{0\}$ ). Corresponding to this decomposition of $W, M$ splits as $M_{0} \oplus M_{1}$-see the remark following Lemma 3.5. Indeed $M_{0}$ is equal to $\gamma\left(C_{G}^{\infty}(V, W)_{o}\right)$. It is easily verified that the subspaces $M_{0}$ and $M_{1}$ are left invariant by $\Omega$ and $\Pi$. That is, $\Omega(g)\left(M_{j}\right) \subset M_{j}$, $j=0,1, g \in C_{G}^{\infty}(V, V)_{o}$ and similarly for $\Pi$ (although $\Omega$ respects the splitting of $M, \Pi$ need not). We let $\Omega_{0}$ and $\Omega_{1}$ denote the restriction of $\Omega$ to $M_{0}$ and $M_{1}$ respectively. Similarly we define $\Pi_{0}$ and $\Pi_{1}$.

The proof of the following lemma is similar to that of Lemma 3.7 and we omit it.

LeMma 3.8. $\Pi_{0}\left(C_{G}^{\infty}(W, W)_{o}\right) \subset P\left(M_{0}, M_{0}\right)$ and $\Pi_{1}\left(C_{G}^{\infty}(W, W)_{o}\right)=$ $C^{\infty}\left(M_{1}, M_{1}\right)_{o}$. Moreover, $\Pi_{0}$ restricts to a group homomorphism of $\operatorname{Diff}_{G}(W)_{o}$ into the group of invertible polynomials. 
Remarks. (1) $\Pi_{0}(h)$ depends only on the germ of $h$ at zero, $h \in$ $C_{G}^{\infty}(W, W)_{0}$.

(2) It may easily be shown that if $f \in \operatorname{Diff}_{G}(W)_{o}$, then $\Pi_{0}(f)=\Pi(D f(0))$. $(I+p)$, where $p$ is a nilpotent polynomial (that is, $p^{r}=0$ for sufficiently large $r$ ). In most simple cases $\Pi_{0}(f)$ will be a linear map for all $f \in$ $C_{G}^{\infty}(W, W)_{o}$.

(3) The above constructions have parametrized analogues which we now briefly describe. Suppose that $U$ is a trivial $G$-space and let

$$
C_{G}^{\infty}(V \times U, V \times U)_{0}
$$

denote the set of $g \in C_{G}^{\infty}(V \times U, V \times U)$ such that $g$ maps $\{0\} \times U$ diffeomorphically onto $\{0\} \times U$. Given

$$
g=\left(g_{1}, g_{2}\right) \in C_{G}^{\infty}(V \times U, V \times U)_{o},
$$

we define $\alpha(g) \in \operatorname{Diff}(U)$ and $\beta(g): U \rightarrow C_{G}^{\infty}(V, V)_{o}$ by $\alpha(g)=g \mid\{0\} \times U$ and

$$
\beta(g)(u)(v)=g_{1}(\operatorname{Id} \times \alpha(g))^{-1}(v, u), \quad u \in U, v \in V .
$$

It may be verified that for all $f \in C_{G}^{\infty}(V \times U, W)_{o}, s \in U$, we have

$$
\gamma(f g)(s)=[\gamma(f)(\alpha(g)(s))] \Omega(\beta(g)(s)) .
$$

In particular, no essentially new transformations of $M$ are obtained by considering parametrized families of maps.

For our applications we shall only be interested in the transformations of $M_{0}$ induced by $\Omega_{0}$ and $\Pi_{0}$. We let

$$
G_{1}=\Pi_{0}\left(\operatorname{Diff}_{G}(V)_{o}\right), \quad G_{2}=\Pi_{o}\left(\operatorname{Diff}_{G}(W)_{o}\right) .
$$

$G_{1}$ and $G_{2}$ are subgroups of the group of invertible polynomial diffeomorphisms of $M_{0}$. Let $Q$ denote the subgroup of $\operatorname{Diff}\left(M_{0}\right)_{o}$ generated by $G_{1}$ and $G_{2}$. Every element of $\mathcal{Q}$ is thus a polynomial diffeomorphism of $M_{0}$. By taking the product of elements of $\mathbb{Q}$ with the identity map on $M_{1}$ we may regard $\mathcal{Q}$ as defining a group of transformations of $M$ and it will often be convenient to do this in the sequel. We shall now give a more geometric interpretation of the groups $G_{1}, G_{2}$ and $Q$.

Let $\left\{F_{1}, \ldots, F_{k}\right\}$ be an MSG for $P_{G}(V, W)$ and take the corresponding basis for $M$. Let $F: V \times M \rightarrow W$ be the equivariant polynomial defined by

$$
F(x, t)=\sum_{j=1}^{k} t_{j} F_{j}(x)
$$

where $\left(t_{1}, \ldots, t_{k}\right)$ are the coordinates of $t \in M$ and we regard $M$ as a trivial $G$-space. Set

$$
X=\{(x, t) \in V \times M: F(x, t)=0\} .
$$


Earlier we verified that a change of MSG corresponded to a linear change of coordinates on $M$. A similar argument shows that a change of MSG induces a polynomial endomorphism of $V \times M$ which preserves $X$ and restricts to a diffeomorphism on some neighbourhood of $\{0\} \times M$ in $V \times M$. Hence the germ of $X$ along $\{0\} \times M$ is uniquely determined up to algebraic isomorphism.

We let $X(t)=\{x \in V: F(x, t)=0\}, t \in M . X(t)=\varnothing$ if $t \notin M_{0}$ and for the rest of this section we shall assume, without loss of generality, that $M=M_{0}$. We also fix the MSG and identify $M$ with $R^{k}$. Let $g \in G_{2}$ and choose $f \in \operatorname{Diff}_{G}(W)_{o}$ such that $\Pi(f)=g$. There exist $q_{j} \in C_{G}^{\infty}\left(V \times R^{k}\right)$ such that

$$
(f F)(x, t)=\sum_{j=1}^{k} q_{j}(x, t) F_{j}(x) .
$$

Set $q=\left(q_{1}, \ldots, q_{k}\right) \in C_{G}^{\infty}\left(V \times R^{k}, R^{k}\right)$. Clearly $g(t)=\Pi(f)(t)=q(0, t)$, $t \in R^{k}$, and the map $(x, t) \mapsto g(t)$ therefore represents a polynomial approximation to $q$ near $\{0\} \times R^{k}$. Now $F(x, t)=0$ if and only if $(f F)(x, t)=$ 0 , that is if and only if $F(x, q(x, t))=0$. Letting $Q$ denote the map $(x, t) \mapsto$ $(x, q(x, t))$, we see immediately that $Q$ maps $X$ into itself. $Q$ restricts to a diffeomorphism on some neighbourhood of $\{0\} \times R^{k}$ and so $(x, t) \mapsto$ $(x, g(t))$ represents a polynomial approximation to a local symmetry of $X$. It will follow from the results given in $\$ 4$ that, off a proper semialgebraic subvariety of $R^{k}$ (always contained in $M_{0}$ ), the germs of $X(t)$ and $X(g t)$ at zero are homeomorphic. In fact rather more is true, but we leave the precise formulation to the next section. Similar results hold for elements of $G_{1}$ and $Q$.

4. Equisingularity and local triviality. In this section we shall use stratification theory to prove the equisingularity and isotopy results that we shall need in $\$ 5$ when we frame our (local) definition of $G$-transversality. We shall assume some familiarity with stratification theory and, in particular, with Whitney's regularity conditions (a) and (b) and Thom's first isotopy lemma. Mather's paper [10] provides an excellent reference here and we shall follow its terminology throughout this section. For background on equisingularity theory we refer to Teissier's survey [15], but see also Varčenko [18].

Let $X$ be a closed semianalytic subset of $R^{m} \times R^{k}[10$, p. 206]. We give $X$ the canonical stratification (that is, the minimal Whitney stratification). We shall denote the union of the $j$-dimensional strata of $X$ by $X_{j} .\left\{X_{j}: 0 \leqslant j \leqslant k\right.$ $+m$ \} will, therefore, be a decomposition of $X$ into mutually disjoint strongly analytic submanifolds of $R^{m} \times R^{k}$. Each pair $\left(X_{j}, X_{s}\right), j>s$, will then satisfy Whitney's regularity conditions (a) and(b). That is, if $x \in \bar{X}_{j} \cap X_{s}$, Whitney's regularity conditions are satisfied at $x$ for the pair $\left(X_{j}, X_{s}\right)$. 
REMARK. Throughout the first part of this section we shall work within the class of semianalytic stratified sets. If $X$ is, in addition, a semialgebraic subset of $R^{m} \times R^{k}$, then all our constructions will result in semialgebraic, rather than just semianalytic, sets. In particular, the canonical stratification of $X$ will be semialgebraic (see [10, p. 211]).

Let $\pi: X \rightarrow R^{k}$ denote the restriction of the projection $R^{m} \times R^{k} \rightarrow R^{k}$ to $X$. For $t \in R^{k}$, we set $X(t)=\pi^{-1}(t)(X(t)$ may be empty). We shall be studying local triviality properties of the map $\pi$ in a neighbourhood of $\{0\} \times R^{k}$ and the type of equisingularity that we shall be studying is essentially "Whitney equisingularity" (see [15]). We define a "hierarchy" of (local) equisingularity types for $\pi$ and show that each type is implied by a certain set of Whitney regularity conditions. We also state various isotopy lemmas which follow, using (a) and (b) regularity, from Thom's first isotopy lemma.

In the next definition, and elsewhere in the sequel, we sometimes regard $R^{k}$ as embedded in $R^{m} \times R^{k}$ as $\{0\} \times R^{k}$.

DEFINITION 4.1. An equisingularity sequence for $\pi$ consists of a sequence $E_{0} \supseteq \cdots \supseteq E_{k}$ of closed semianalytic subsets of $R^{k}$ satisfying

(1) $E_{0}=R^{k}$ and for $q=0, \ldots, k, E_{q} \backslash E_{q+1}$ is a, possibly empty, semianalytic submanifold of $R^{k}$ of codimension $q$.

(2) $\left\{X_{j} \backslash E_{0}, E_{q} \backslash E_{q+1}: 0 \leqslant j \leqslant k+m, 0 \leqslant q \leqslant k\right\}$ is a Whitney stratification of $X \cup R^{k}$.

(3) $X_{j} \cap\left(E_{q} \backslash E_{q+1}\right)$ is empty for $j<k-q$ and is an open (possibly empty) subset of $E_{q} \backslash E_{q+1}$ for $j=k-q$.

REMARK. Notice that the pair $\left(X_{i} \backslash E_{0}, X_{j} \backslash E_{0}\right), i>j \geqslant 0$, automatically satisfies Whitney's regularity conditions.

The following simple example illustrates condition (3) further.

EXAMPLE. Let $X$ be a closed semianalytic subset of $R^{k} \subset R^{m} \times R^{k}$ with canonical stratification $\left\{X_{j}: 0 \leqslant j \leqslant k\right\}$. An equisingularity sequence for $\pi$ is given by

$$
E_{0}=R^{k} \text { and } E_{k-s}=\bigcup_{j \leqslant s} X_{j}, \quad 0 \leqslant s \leqslant k .
$$

DEFINITION 4.2. A fundamental equisingularity sequence for $\pi$ (an "FES") is an equisingularity sequence $A_{0} \supseteq \cdots \supseteq A_{k}$ which satisfies the additional condition

"If $B_{0} \supseteq \cdots \supseteq B_{k}$ is any equisingularity sequence for $\pi$, then either $B_{j}=A_{j}, 0 \leqslant j \leqslant k$, or there exists $j_{0}$, $0 \leqslant j_{0} \leqslant k$, such that

$$
\begin{gathered}
B_{j}=A_{j}, \quad j<j_{0}, \\
B_{j_{0}} \text { strictly contains } A_{j_{0}}, "
\end{gathered}
$$


THEOREM 4.3. A fundamental equisingularity sequence for $\pi$ exists. It is unique.

Proof. Take $A_{0}=R^{k}$ and define

$$
A_{0} \backslash A_{1}=\left(R^{k} \backslash X\right) \cup \bigcup_{j>0} \operatorname{Interior}\left(X_{j} \cap R^{k}\right),
$$

where the interiors are taken in $R^{k}$. Clearly the conditions of Definition 4.1 hold, where applicable (notice that if $x \in \operatorname{Interior}\left(X_{j} \cap R^{k}\right)$, then $x$ does not lie in the closure of any stratum of dimension less than $j$ ). The proof now proceeds by induction. Suppose that we have constructed $A_{0}, \ldots, A_{q}, q<k$, so that the conditions of Definition 4.1 hold, where applicable. If $A_{q}$ is of codimension greater than $q$, we set $A_{q+1}=A_{q}$ (notice that condition (3) of Definition 4.1 continues to hold). Suppose codimension $\left(A_{q}\right)=q$. We claim that there exists a (minimal) closed semianalytic subset $A_{q+1}^{\prime}$ of $A_{q}$ such that the following hold:

(1) codimension $\left(A_{q+1}^{\prime}\right)>q$.

(2) $A_{q} \backslash A_{q+1}^{\prime}$ is an open subset of the set of regular points of $A_{q}$.

(3) The strata $A_{p} \backslash A_{p+1}, 0 \leqslant p \leqslant q-1, A_{q} \backslash A_{q+1}^{\prime}$ together form a Whitney stratification of $A_{0} \backslash A_{q+1}^{\prime}$.

The proof of the existence of $A_{q+1}^{\prime}$ follows, using Lojasiewicz' results on semianalytic sets [8], exactly the construction of minimal Whitney stratifications given by Mather in [10, pp. 210-211], and we shall not repeat the details here. We define

$$
K_{q}=\bigcup_{j>0} \operatorname{Interior}\left(\left(A_{q} \backslash A_{q+1}^{\prime}\right) \cap X_{j}\right),
$$

where the interiors are taken in $A_{q} \backslash A_{q+1}^{\prime}$. Finally we set

$$
A_{q+1}=A_{q+1}^{\prime} \cup\left(A_{q} \backslash K_{q}\right) \text {. }
$$

It is easily verified that $A_{0} \supseteq \cdots \supseteq A_{q+1}$ satisfies the conditions of Definition 4.1, where applicable, and so the inductive step is completed. Finally, the equisingularity sequence that we have constructed is clearly an FES (cf. the proof of the existence of a minimum stratification given in [10]).

Definition 4.4. Let $A_{0} \supseteq \cdots \supseteq A_{k}$ be the FES for $\pi$. We say that a point $t \in R^{k}$ is a point of $q$-Whitney equisingularity for $\pi$ if $t \in A_{q} \backslash A_{q+1}$.

Suppose that $t_{0} \in R^{k}$ is a point of $q$-Whitney equisingularity for $\pi$. Making a local change of coordinates, we may assume that $A_{q} \backslash A_{q+1}=\{0\} \times R^{k-q}$ and that $t_{0}=(0,0)$. For each $w \in A_{q} \backslash A_{q+1}$, we let $Q_{w}=R^{q} \times\{w\}$ and $X_{w}=\pi^{-1}\left(Q_{w}\right)$. With these assumptions and notation we have

LEMMA 4.5. If $t_{0}$ is a point of $q$-Whitney equisingularity for $\pi$, we may find open neighbourhoods $W$ of $t_{0}$ in $A_{q} \backslash A_{q+1}, Z$ of $\left(0, t_{0}\right)$ in $R^{m} \times R^{k}, V_{0}$ of 0 in $R^{q}$ and a homeomorphism 


$$
\Phi: Z_{0} \times W \rightarrow Z
$$

where $Z_{0}=Z \cap\left(R^{m} \times V_{0}\right)$, such that

(1) $\Phi$ covers the identity map on $W$, restricts to the identity map on $W$ and $\Phi(0, t) \in\{0\} \times R^{k}$, for all $t \in R^{k}$.

(2) $\Phi\left(\left(X_{0} \cap Z_{0}\right) \times\{w\}\right)=X_{w} \cap Z$, for all $w \in W$.

(3) For fixed $z, w \mapsto \Phi(z, w)$ is $C^{\infty}$.

Proof. The result is a well-known corollary of Thom's first isotopy lemma (see [10] and also [16, p. 270]). Notice that the conditions on the FES imply that there exists a neighbourhood of $t_{0}$ in $R^{m} \times R^{k}$ which does not meet any strata of $X$ of dimension less than $k-q$. The map $\Phi$ also preserves stratifications.

Definition 4.6. Let $B$ be a codimension $q$ submanifold of $R^{k}$ and suppose that $f: R^{m} \times R^{s} \rightarrow R^{m} \times R^{k}$ is $C^{\infty}$ and that $f\left(\{0\} \times R^{s}\right) \subset\{0\} \times R^{k}$. We say that $f$ is $q$-admissible at $s \in R^{s}$, relative to $B$, if $f$ is transversal to $\{0\} \times R^{k}$ and one of the following conditions holds:

(1) $f(s) \notin B$.

(2) $f(s) \in B$ and $f$ is transverse to $B$ at $s$.

If $f$ is $q$-admissible at every point of $R^{s}$, we shall say that $f$ is $q$-admissible.

REMARKs. (1) If $f$ is $q$-admissible at $s$ and $f(s) \in B$, then $f \mid\{0\} \times R^{s}$ is transverse to $B \subset R^{k}$ at $s$.

(2) If $f$ is $q$-admissible at $s$, it is $q$-admissible on some nieghbourhood of $s$ in $R^{s}$.

We now take $B=A_{q} \backslash A_{q+1}$ and $s_{0} \in B$. By a local change of coordinates we may assume that $B=\{0\} \times R^{k-q}$ and $s_{0}=(0,0)$. Set $R^{s}=R^{q} \times R^{s-q}$, $R^{k}=R^{q} \times R^{k-q}$. Let $f=\left(f_{1}, f_{2}\right): R^{m} \times R^{s} \rightarrow R^{m} \times R^{k}$ be 0 -admissible relative to $\{0\} \times R^{k}$ and suppose that there exists a $C^{\infty} \operatorname{map} g: R^{s} \rightarrow R^{k-q}$ such that

$$
f_{2}\left(0, s_{1}, s_{2}\right)=\left(s_{1}, g\left(s_{1}, s_{2}\right)\right) \text { for all }\left(s_{1}, s_{2}\right) \in R^{q} \times R^{q-s} .
$$

$f$ is obviously $q$-admissible, relative to $B$. Moreover, by a local change of coordinates in $R^{s}$, any map $f$ which is $q$-admissible at $s_{0}, f\left(s_{0}\right) \in B$, can be represented locally by a function of the above type. With these assumptions on $B=A_{q} \backslash A_{q+1}$ and $f$, we have

THEOREM 4.7. Suppose that $0 \in A_{q} \backslash A_{q+1}, f: R^{m} \times R^{s} \rightarrow R^{m} \times R^{k}$ is $q$-admissible at 0 and $f(0)=0$. Then there exists an open neighbourhood $W$ of 0 in $R^{s-q}$, an open neighbourhood $D$ of $(0,0)$ in $R^{m} \times R^{s}$ and a homeomorphism

$$
\Gamma_{f}: D_{0} \times W \rightarrow D
$$

where $D_{0}=D \cap\left(R^{m} \times R^{q} \times\{0\}\right)$, such that

(1) $\Gamma_{f}$ covers the identity map on $W$ and $\Gamma_{f}(0, w)=(0, w)$, for all $w \in W$.

(2) If we set $\tilde{X}_{w}=f^{-1}(X) \cap\left(R^{m} \times R^{q} \times\{w\}\right) \cap D$, then 


$$
\Gamma_{f}\left(\tilde{X}_{0} \times\{w\}\right)=\tilde{X}_{w} \text { for all } w \in W .
$$

Proof. An application of Thom's first isotopy lemma [16, p. 270].

The following result, which we make much use of in later sections, also follows from Thom's first isotopy lemma.

THEOREM 4.8. Suppose that $f: R^{m} \times R^{s} \rightarrow R^{m} \times R^{k}$ is q-admissible and that $f\left(0, s_{0}\right) \in A_{q} \backslash A_{q+1}$ is a point of $q$-Whitney equisingularity for $\pi$. We may find an open relatively compact neighbourhood $M \times S$ of $\left(0, s_{0}\right)$ in $R^{m} \times R^{s}$ and $\varepsilon>0$, such that if $g: R^{m} \times R^{s} \rightarrow R^{m} \times R^{s}$ is q-admissible, equal to $f$ outside of some compact subset $K \subset M \times S$ and is within $\varepsilon$ of $f$ in the $C^{1}$ norm on $M \times S$, then there exists an isotopy

$$
H:\left(R^{m} \times R^{s}\right) \times[0,1] \rightarrow R^{m} \times R^{s}
$$

satisfying the following conditions:

(1) $H_{t}=H \mid\left(R^{m} \times R^{s}\right) \times\{t\}$ is a homeomorphism for all $t \in[0,1]$.

(2) $H_{t}$ is equal to the identity map on $\left(R^{m} \times R^{s}\right) \backslash K$, for all $t \in[0,1]$.

(3) $H_{0}$ is the identity map and $H_{1}\left(g^{-1}(X)\right)=f^{-1}(X)$.

(4) $H_{t}\left(\{0\} \times R^{s}\right) \subset\{0\} \times R^{s}$, for all $t \in[0,1]$.

(5) For fixed $f, H_{t}$ depends continuously on $g$ and tends to the identity map uniformly in $t$ as $g$ tends to $f\left(C^{1}\right.$ topology).

Suppose that $G$ is a compact Lie group and that $V$ and $W$ are linear $G$-spaces. As described in $\S 3$, we may associate to $V$ and $W$ a (unique) algebraic subvariety $X$ of $V \times R^{k}$. $X$ is given explicitly as the zero locus of $\sum_{j=1}^{k} t_{j} F_{j}$, where $\left\{F_{1}, \ldots, F_{k}\right\}$ is an MSG for $P_{G}(V, W)$. As above we let $\pi$ denote the restriction of the projection map $V \times R^{k} \rightarrow R^{k}$ to $X$ and give $X$ its canonical stratification. As we remarked earlier, this stratification is semialgebraic.

Definition 4.9. The fundamental equisingularity sequence of the (ordered) pair of $G$-spaces $(V, W)$ is defined to be the fundamental equisingularity sequence of $\pi$.

REMARKS. (1) The FES of $(V, W)$ depends only on the isomorphism classes of the $G$-representation spaces $V$ and $W$.

(2) If $G$ acts trivially on $R^{s}$, then the FES's of $(V, W)$ and $\left(V \times R^{s}, W\right)$ are equal. This is immediate from Lemma 3.5.

(3) Recall from $\$ 3$ that we have a Lie group $\mathbb{Q}$ of transformations of $R^{k}$ associated to $V$ and $W$. We claim that if $A_{0} \supseteq \cdots \supseteq A_{k}$ is the FES for $(V, W)$ then it is left invariant by $Q$. That is, $g\left(A_{q}\right)=A_{q}, q \geqslant 0$ and all $g \in \mathbb{Q}$. This is an immediate consequence of the definition of the FES and the discussion at the end of $\S 3$. We also note that since $A_{1} \subset \gamma\left(C_{G}^{\infty}(V, W)_{o}\right)$ $=M_{0}$, whether or not $W$ contains the trivial representation, the FES is left 
invariant by the group of diffeomorphisms of $R^{k}$ generated by $\Omega\left(\operatorname{Diff}_{G}(V)_{o}\right)$ and $\Pi\left(\operatorname{Diff}_{G}(W)_{o}\right)$.

(4) In an earlier version of this paper we defined an equisingularity sequence to be a sequence $E_{0} \supseteq \cdots \supseteq E_{k}$ of algebraic subvarieties of $R^{k}$ satisfying the conditions of Definition 4.1 (it was assumed that $X$ was algebraic). After constructing such a sequence, using a variant of the proof of Theorem 4.3, the existence of an (algebraic) FES then followed from the fact that arbitrary intersections of algebraic varieties are algebraic (see also [5]).

5. Local models for $G$-transversality. For this section we shall suppose that we are given fixed linear $G$-spaces $V, W_{1}$ and $W_{2} . V$ decomposes uniquely as a direct sum of linear $G$-spaces, $\tilde{V} \oplus U$, where $U$ is a trivial $G$-space and $G$ does not act trivially on any proper subspace of $\tilde{V}$. We shall always assume that $U$ is associated to $V$ in this way and regard $U$ as a vector subspace of $V$. Fix an MSG for $P_{G}\left(\tilde{V}, W_{2}\right)$ and corresponding FES $A_{0} \supseteq \cdots \supseteq A_{k}$ for $\left(\tilde{V}, W_{2}\right)$. Denote this FES by $\mathscr{F}$. By Remark 2 at the end of $\S 4, \mathscr{F}$ is also an FES for $\left(V, W_{2}\right)$. Given any $f=\left(f_{1}, f_{2}\right) \in C_{G}^{\infty}\left(V, W_{1} \times W_{2}\right)$, we have a $C^{\infty}$ map $\gamma\left(f_{2}\right): U \rightarrow R^{k}$ (Lemma 3.6).

Suppose that $g: R^{s} \rightarrow R^{k}$. We shall say that $g$ is transverse to $\mathscr{F}$ if $g$ is transverse to each submanifold $A_{j} \backslash A_{j+1}$, that is, if $g$ is transverse to the Whitney stratification of $R^{k}$ associated to the FES.

Definition 5.1. Let $f=\left(f_{1}, f_{2}\right) \in C_{G}^{\infty}\left(V, W_{1} \times W_{2}\right)$. We say that $f$ is $G$-transversal to $W_{1} \times\{0\}$ at $s \in U$ if either $f(s) \notin W_{1} \times\{0\}$ or $f(s) \in W_{1}$ $\times\{0\}$ and $\gamma\left(f_{2}\right): U \rightarrow R^{k}$ is transverse to $\mathscr{F}$ at $s$. We say that $f$ is $G$-transversal to $W_{1} \times\{0\}$ on $U$ if it is $G$-transversal to $W_{1} \times\{0\}$ at every point on $U$.

REMARK. Let $F_{j}$ be the vector subspace of $W_{j}$ on which $G$ acts trivially, $j=1,2 . f(U) \subset F_{1} \times F_{2}$ and it is easily verified that if $f$ is $G$-transversal to $W_{1}$ on $U$ then $f \mid U$ is transversal to $F_{1}$ in $F_{1} \times F_{2}$. That is, $G$-transversality implies weak $G$-transversality.

We now prove some local results that we need in $\S 6$. We let $C_{G}^{\infty}\left(V, W_{1} \times\right.$ $\left.W_{2} ; r\right)$ denote the space $C_{G}^{\infty}\left(V, W_{1} \times W_{2}\right)$ with the (Whitney) $C^{r}$-topology. We remark that if $f, g \in C_{G}^{\infty}\left(V, W_{1} \times W_{2}\right)$ are equal outside a compact subset of $V$, they are close in the Whitney $C^{r}$-topology if and only if they are close in the $C^{r}$ - (metric) topology. We let $d$ denote the maximum degree of the polynomials for the given MSG of $P_{G}\left(V, W_{2}\right)(d$ is independent of the choice of MSG; see \$3).

LeMma 5.2. Let $K$ be a compact subset of $U$ and suppose that $f \in C_{G}^{\infty}\left(V, W_{1}\right.$ $\left.\times W_{2}\right)$ is $G$-transversal to $W_{1} \times\{0\}$ on $K$. Then there exists an open neighbourhood $N(K, f)$ of $f$ in $C_{G}^{\infty}\left(V, W_{1} \times W_{2} ; d+1\right)$ such that every $g \in N(K, f)$ is G-transversal to $W_{1} \times\{0\}$ on $K$. 
Proof. Immediate from Lemma 3.6 and the openness of transversal intersection.

Lemma 5.3. Suppose that $f \in C_{G}^{\infty}\left(V, W_{1} \times W_{2}\right)$ is $G$-transversal to $W_{1} \times$ $\{0\}$ at $0 \in V$. Then there exists a $G$-invariant relatively compact open neighbourhood $Q$ of 0 in $V$ and a neighbourhood $N(f)$ of $f$ in $C_{G}^{\infty}\left(V, W_{1} \times W_{2}\right.$; $d+1)$, such that if $g \in N(f)$ is equal to $f$ outside of $Q$, then there exists an equivariant isotopy $H: V \times[0,1] \rightarrow V$ satisfying

(1) $H_{t}=$ identity map on $V \backslash Q$, for all $t \in[0,1]$.

(2) $H_{0}=$ identity map on $V$.

(3) $H_{1}\left(g^{-1}\left(W_{2} \times\{0\}\right)\right)=f^{-1}\left(W_{1} \times\{0\}\right)$.

(4) For fixed $f, H_{t}$ depends continuously on $g$ and tends to the identity map uniformly in $t$ as $g$ tends to $f\left(C^{d+1}\right.$-topology).

Proof. $f=\left(f_{1}, f_{2}\right)$ is $G$-transversal to $W_{1} \times\{0\}$ if and only if $f_{2}$ is $G$-transversal to $0 \in W_{2}$. It is therefore no loss of generality to take $W_{1}=$ $\{0\}$ in the statement of the lemma. Set $W_{2}=W$, write $V=\tilde{V} \oplus U$ and take coordinates $(x, s)$ on $\tilde{V} \oplus U$. Let $\left\{F_{1}, \ldots, F_{k}\right\}$ be an MSG for $P_{G}(V, W)$. Given $h \in C_{G}^{\infty}(V, W)$, there exist $q_{1}, \ldots, q_{k} \in C_{G}^{\infty}(V)$ such that

$$
h(x, s)=\sum_{j=1}^{k} q_{j}(x, s) F_{j}(x) .
$$

We let $Q(h): \tilde{V} \oplus U \rightarrow \tilde{V} \times R^{k}$ denote the map

$$
(x, s) \mapsto\left(x,\left(q_{1}(x, s), \ldots, q_{k}(x, s)\right)\right) .
$$

Observe that $Q(h) \mid U=\gamma(h)$. Since the lemma is trivial if $f(0) \neq 0$, we suppose $f(0)=0$. If $\gamma(f)(0) \in A_{q} \backslash A_{q+1}, Q(f)$ is $q$-admissible at zero. All the statements of the lemma, save the equivariance of the isotopy, now follow easily from Theorem 4.8 and Lemma 3.6. To prove that $H$ is equivariant, we first observe that the variety $X$ and its canonical stratification are $G$-invariant (trivial action of $G$ on $R^{k}$ ). A simple check of Mather's proof of Thom's first isotopy lemma shows that the vector fields used in the construction of the isotopy may be taken to be equivariant. We omit details, as this type of argument is standard in equivariant topology.

REMARK. Theorem 4.7 may be used to give "local models" for $G$-transversal intersections. For example, suppose that $f \in C_{G}^{\infty}(V, W)$ is $G$-transversal to $0 \in W, f(0)=0$ and $\gamma(f)(0) \in A_{q} \backslash A_{q+1}$. Let $D$ be a (small) $q$-dimensional disc in $R^{k}$ with centre $\gamma(f)(0)$ and transverse to $A_{q} \backslash A_{q+1}$. Theorem 4.7 implies that, near zero, $f^{-1}(0)$ may be parametrized as a family of varieties locally isotopic to a trivial family of sections of $X$ of the form $\pi^{-1}(D)$.

ExAmple. Let $f: C \rightarrow C$ be a $C^{\infty} S^{1}$-invariant map, where $S^{1}$ acts as $z \mapsto e^{p i \theta_{z}}$ on the domain of $f$ and as $z \mapsto e^{q i \theta_{z}}$ on the range of $f$ and $p$ and $q$ are nonzero positive integers (so implying $f(0)=0$ ). We consider the $S^{1}$. 
transversality of $f$ to $0 \in C$. Any $S^{1}$-invariant (real) linear map may be written in the form $z \mapsto a z, a \in C$. In case $p \neq q, a=0$ (Schur's lemma). Since $D f(0)$ is $S^{1}$-invariant, we can never have $f$ transversal to 0 at 0 if $p \neq q$. In case $p=q$, we can always perturb $f$ to be transversal to zero (cf. §2). Assume $p \neq q$. For all $z \in C$, the isotropy group of $S^{1}$ at $f(z)$ contains that of $S^{1}$ at $z$ (since $g(z)=z$ implies $g f(z)=f(g z)=f(z)$ ). Hence if $z$ and $f(z)$ are nonzero, $Z_{p} \subset Z_{q}$. But this can only happen if $p$ divides $q$. If $p$ does not divide $q, f$ must vanish identically and the $S^{1}$-transversality of $f$ to zero always holds. Since, in this case, $f(C)=\{0\}$, the intersection is highly stable! Suppose $q=p k, k \neq 1$. Then an MSG for $P_{S^{\prime}}(C, C)$ is given by the polynomials $z^{k}$ and $i z^{k}$ and the corresponding FES is given by $A_{0}=R^{2}, A_{1}=A_{2}$ $=\{0\} . f$ may be written in the form

$$
f(z)=p_{1}(z) z^{k}+p_{2}(z) i z^{k},
$$

where $p_{1}$ and $p_{2}$ are real valued $S^{1}$-invariant $C^{\infty}$ functions on $C$. $f$ is $S^{1}$-transversal to 0 at 0 if one of $p_{1}(0), p_{2}(0)$ is nonzero. If this condition holds, the intersection of $f$ with zero is isolated and equal to zero.

It may be helpful to interpret the above results in the following way. Let $\operatorname{graph}(f): C \rightarrow C \oplus C$ denote the graph map of $f$ and take the $S^{1}$-action $\left(z_{1}, z_{2}\right) \mapsto\left(e^{p i \theta} z_{1}, e^{q i \theta} z_{2}\right)$ on $C \oplus C$. graph $(f)$ is $S^{1}$-transversal to the $z_{1}$-axis at zero if and only if $f$ is $S^{1}$-transversal to zero at zero. In case $p$ does not divide $q$, $\operatorname{graph}(f)=z_{1}$-axis. If $q=p k, k \neq 1, \operatorname{graph}(f)$ is always tangent to the $z_{1}$-axis at zero. $S^{1}$-transversality of $f$ to zero implies, in this case, that the intersection is isolated and stable. Notice that the cases $p=q, p$ divides $q$ and $p$ does not divide $q$ correspond respectively to the $S^{1}$-action on $C \oplus C$ having 2, 3 or 4 orbit types. In particular, the more orbit types an $S^{1}$-action has, the less chance there is of being able to achieve transversality on the fixed point set (cf. [12]).

6. Transversality in $G$-manifolds. I: The isotopy theorem. In this section we define $G$-transversality for the category of $G$-manifolds and prove an isotopy theorem for $G$-transversal intersections. To establish notation, we first review a little of the elementary theory of differentiable Lie group actions (for a more complete introduction we refer to Palais' lecture in [11] or Bredon's text [2]). All manifolds will be assumed $C^{\infty}$ and without boundary; all Lie group actions $C^{\infty}$.

Suppose that $G$ is a compact Lie group acting on the manifold $M$. For each $x \in M$, we define the isotropy subgroup of $G$ at $x$ by

$$
G_{x}=\{g \in G: g(x)=x\} \text {. }
$$

$G_{x}$ is a closed subgroup of $G$ and the $G$-orbit through $x, G(x)$, is equivariantly diffeomorphic to the homogeneous space $G / G_{x}, x, y \in M$ are said to 
be of the same orbit type if $G(x)$ is equivariantly diffeomorphic to $G(y)$ or, equivalently, if $G_{x}$ and $G_{y}$ are conjugate subgroups of $G$. "Orbit type" defines an equivalence relation on points of $M$. We let $\left\{M_{i}: i \in I\right\}$ denote the corresponding partition of $M$ into points of the same orbit type. Whilst the sets $M_{i}$ are all submanifolds of $M$, they need not be closed, connected or of constant dimension. However, this partition of $M$ is locally finite and so, if $M$ is compact, only a finite number of orbit types can occur.

We define a partial order $\leqslant$ on the indexing set $I$ by $i \leqslant j$ if there exist $x \in M_{i}$ and $y \in M_{j}$ such that $G_{x} \supseteq G_{y}$. We remark that if $\partial M_{j} \cap M_{i} \neq \varnothing$, then $i<j$ (the converse need not be true). Given any compact subset $K$ of $M$ and $i \in I$, the set $\cup_{j<i} M_{j} \cap K$ is always closed.

Given $i \in I$, we may decompose $M_{i}$ uniquely as a union of connected submanifolds of $M$ :

$$
M_{i}=\bigcup_{j \in J(i)} M_{i}^{j}
$$

As we remarked above, the dimension of $M_{i}^{j}$ may vary with $j$. However, the family $\left\{M_{i}^{j}: j \in J(i)\right\}$ is locally finite for all $i \in I$.

We recall the following version of the differentiable slice theorem: "Given $x \in M$ and a $G$-invariant open neighbourhood $V$ of $x$, we may find a $G$-invariant tubular neighbourhood $U \subset V$ of $G(x)$ which is equivariantly diffeomorphic to $G \times_{G_{x}} S_{x}$, where $S_{x}$ is the fibre of the normal bundle to $G(x)$ at $x . " S_{x}$ is a linear $G_{x}$-space. In what follows we frequently identify $U$ with $G \times{ }_{G_{x}} S_{x} . S_{x}$ is then embedded as a disc, centre $x$, transverse to $G(x)$. An equivariant map defined on $U$ is uniquely determined by its restriction to $S_{x}$.

If $N$ is also a $G$-manifold, we let $C_{G}^{\infty}(M, N)$ denote the space of equivariant $C^{\infty}$ maps from $M$ to $N$ and $C_{G}^{\infty}(M, N ; r)$ denote this space with the Whitney $C^{r}$-topology.

If $f \in C_{G}^{\infty}(M, N)$ and $x \in M$ then clearly $G_{x} \subset G_{f(x)}$. Suppose that $W$ is a $G$-invariant submanifold of $N$ and that $f(x) \in W$. We may choose a $G_{x}$-invariant diffeomorphism $\phi$ of a neighbourhood $V$ of $f(x)$ in $N$ with a linear $G_{x}$-space $W_{1} \times W_{2}$ in such a manner that $W$ corresponds to $W_{1} \times\{0\}$. We call the pair $(V, \phi)$ a " $G_{x}$-chart for $W$ at $f(x)$ ". Hence, by making a choice of slice $S_{x}$ at $x$ and $G_{x}$-chart at $f(x), f$ determines a $C^{\infty} G_{x}$-invariant map $\tilde{f}$ : $S_{x} \rightarrow W_{1} \times W_{2}$. Since $S_{x}$ and $W_{1} \times W_{2}$ are linear $G_{x}$-spaces we can now give

Definition 6.1. Let $M$ and $N$ be $G$-manifolds, $W$ be a $G$-invariant submanifold of $N$ and $f \in C_{G}^{\infty}(M, N)$. We say that $f$ is $G$-transversal to $W$ at $x \in M$ if either $f(x) \notin W$ or $f(x) \in W$ and for some choice of slice $S_{x}$ at $x$ and $G_{x}$-chart for $W$ at $f(x)$, the local representative $\tilde{f}: S_{x} \rightarrow W_{1} \times W_{2}$ is $G_{x}$-transversal to $W_{1} \times\{0\}$ at $x$ in the sense of Definition 5.1. We say $f$ is 
$G$-transversal to $W$, written $f \pitchfork_{G} W$, if $f$ is $G$-transversal to $W$ at every point of $M$.

REMARKS. Our definition of $G$-transversality is independent of the choice of slice at $x$ or $G_{x}$-chart for $W$ at $f(x)$. This is an immediate consequence of the fact that our FES's are left invariant by the group $\mathcal{Q}$ discussed in $\$ 3$ (see Remark 2 following Lemma 3.8 and Remark 3 following Definition 4.9). We also note that if $f$ is $G$-transversal to $W$ at $x$, it is $G$-transversal to $W$ at $g(x)$ for all $g \in G$.

Let $M$ and $N$ be $G$-manifolds and $W$ be a $G$-invariant submanifold of $N$. If $x \in M, y \in W$ and $G_{x} \subseteq G_{y}$, we let $d_{x y}$ denote the maximum degree of an MSG for $P_{G_{x}}\left(S_{x}, N_{y}\right)$, where $S_{x}$ is a slice at $x$ and $N_{y}$ is the fibre at $y$ of a $\left(G_{x}\right)$-normal bundle to $W$. Suppose that $x \in M_{i}, i \in I . d_{x y}$ clearly remains constant on connected components of $\left(M_{i}\right)_{G_{x}} \times W_{G_{x}}$ (the subscript $G_{x}$ denotes the fixed point set of the induced $G_{x}$-action). In case $M$ and $W$ are compact, this set has only finitely many components for each $i \in I . d_{g x, g y}=$ $d_{x, y}$ for all $g \in G$. We let $d$ denote the maximum of $d_{x y}-1$ over all pairs $(x, y) \in M \times W$ such that $G_{x} \supseteq G_{y}$. If $M$ and $W$ are compact, $d$ is finite by the above remark.

Theorem 6.2 (EQuivariant Isotopy TheOREM). Let $M$ and $N$ be $G$-manifolds, $W$ be a closed $G$-invariant submanifold of $N$ and $d$ be as defined above. Suppose that $f \in C_{G}^{\infty}(M, N)$ is $G$-transversal to $W$. Then, for $r \geqslant d$, there exists an open neighbourhood $V$ of $f$ in $C_{G}^{\infty}(M, N ; r)$ such that $g^{-1}(W)$ is continuously equivariantly isotopic to $f^{-1}(W)$ for all $g \in V$. That is, if $g \in V$, there exists a continuous map $\phi: M \times[0,1] \rightarrow M$ such that $\phi_{t}=\phi \mid M \times\{t\}$ is an equivariant homeomorphism for all $t \in[0,1], \phi_{0}$ is the identity map and $\phi_{1}\left(g^{-1}(W)\right)=f^{-1}(W)$.

PROof. We first prove three lemmas.

Lemma A. Let $K$ be a compact subset of $M$ contained in some orbit type $M_{i}$. Suppose that $h \in C_{G}^{\infty}(M, N)$ is $G$-transversal to $W$ on $K$. Then there exists an open neighbourhood $V$ of $h$ in $C_{G}^{\infty}(M, N ; r)$ such that every $g \in V$ is $G$-transversal to $W$ on $K$.

Proof. The result follows easily from Lemma 5.2 by taking a finite cover of $K$ by $G$-invariant tubular neighbourhoods and corresponding set of equivariant charts for $W$.

Lemma B. Suppose that $h \in C_{G}^{\infty}(M, N)$ is G-transversal to $W$ at $x \in M$. We may find a $G$-invariant tubular neighbourhood $U$ of $G(x)$ in $M$ and $a$ neighbourhood $N_{x}(h)$ of $h$ in $C_{G}^{\infty}(M, N ; r)$ such that if $g \in N_{x}(h)$ is equal to $h$ outside of $U$, then there exists an equivariant isotopy $\phi: M \times[0,1] \rightarrow M$ 
satisfying

(1) $\phi_{t}=$ identity map on $M \backslash U$.

(2) $\phi_{0}=$ identity map.

(3) $\phi_{1}\left(g^{-1}(W)\right)=h^{-1}(W)$.

(4) $\phi_{t}$ depends continuously on $g$ and tends to the identity, uniformly in $t$, as $g$ tends to $h$ ( $C^{r}$-topology).

Proof. Similar to that of Lemma A using instead Lemma 5.3. We work on a slice $S_{x}$ and extend the $G_{x}$-invariant isotopy we obtain on $S_{s}$ to a $G$-invariant isotopy on the tubular neighbourhood $G\left(S_{x}\right)$.

Once we have Lemmas $\mathrm{A}$ and $\mathrm{B}$, we may use standard techniques of differential topology to obtain

Lemma C. Let $K$ be a compact subset of $M_{i} \subset M$. Suppose that $h \in$ $C_{G}^{\infty}(M, N)$ is $G$-transversal to $W$ on $K$. Then we may find a $G$-invariant open neighbourhood $U$ of $K$ in $M$ and a neighbourhood $N_{K}(h)$ of $h$ in $C_{G}^{\infty}(M, N ; r)$ such that if $g \in N_{K}(h)$ is equal to $h$ outside of $U$, there exists an equivariant isotopy $\phi: M \times[0,1] \rightarrow M$ satisfying conditions 1 to 4 of Lemma B. Moreover, we may assume that $g$ is $G$-transversal to $W$ on $K$ for all $g \in N_{K}(h)$.

Once we have Lemma $\mathrm{C}$ the proof of the main theorem goes fairly straightforwardly by an induction on orbit type. Let us start by assuming that $M$ is compact. Then there exists a minimal orbit type of $M$, say $M_{1}$, and $M_{1}$ is a compact submanifold of $M$. Hence we may apply Lemma $\mathrm{C}$ to obtain a tubular neighbourhood $U_{1}$ of $M_{1}$ in $M$ and open neighbourhood $N_{1}$ of $f$ in $C_{G}^{\infty}(M, N ; r)$ such that if $h \in N_{1}$ is equal to $f$ outside of $U_{1}$, then $h^{-1}(W)$ is equivariantly isotopic to $f^{-1}(W)$ by an isotopy supported in $U_{1}$. At the $j$ th stage of the induction, we have an open neighbourhood $U_{j}$ of the (closed) set $M_{1} \cup \cdots \cup M_{j}$ and an open neighbourhood $N_{j}$ of $f$ in $C_{G}^{\infty}(M, N ; r)$ such that if $h \in N_{j}$ is equal to $f$ outside of $U_{j}$, then $h^{-1}(W)$ is equivariantly isotopic to $f^{-1}(W)$ by an isotopy supported in $U_{j}$. To achieve the inductive step, we make use of the fact that if $U$ is any open neighbourhood of $M_{1} \cup \cdots \cup M_{j}$, then $M_{j+1} \backslash U$ is compact. (The subscripts are chosen so that order type is preserved, that is $\partial M_{j+1} \subset M_{1} \cup \cdots \cup M_{j}$. Of course, $M_{j+1}$ need not be a closed subset of $M$.) Using Lemma C, together with the usual smoothing techniques, we can then make the inductive step. If $M$ is noncompact, we take a locally finite countable cover of $M$ by compact sets. Since any compact set meets only finitely many orbit types of $M$ we may essentially repeat the above argument. We remark that it is easily seen that the neighbourhood of $f$ we obtain will be open in the Whitney $C^{r}$-topology.

REMARKs. (1) We have not shown that our definitions imply that $G$-transversality is open in the $C^{r}$-topology. Fortunately Lemma $\mathrm{A}$ was all that we needed by way of openness for the proof of the isotopy theorem. 
(2) E. Bierstone has also developed a theory of "equivariant general position" [2a]. He proves that his definition of equivariant transversality is open. The relation between the two definitions is not, at present, completely clear. However, the definition we have given in this paper is, a priori, stronger than that of Bierstone. Added in proof. The definitions are equivalent [20]. One feature of our definition is that the FES's determine the local intersection type of $G$-transversal intersections. Thus, with the notation of $\S 5$, if $f: V \rightarrow W$ is $G$-transversal to $\{0\}$ at 0 , then the germ of $f^{-1}(0)$ at 0 is uniquely determined by the germ of $\pi^{-1}(\gamma(f)(0))$ at zero. We intend to pursue the question of the openness of our definition elsewhere.

EXAMPLE. We cannot, in general, require differential stability in the equivariant isotopy theorem and we present here a simple counterexample. Let $S^{1}$ act on $C$ and $C \oplus C$ as scalar multiplication by $e^{4 i \theta}$ and $e^{i \theta}$ respectively. An MSG for $P_{S^{1}}(C \oplus C, C)$ is given by the set of homogeneous monomials of degree four with coefficients +1 and $+i$ (see $\S 5$ ). Working with complex coefficients, we see that the general $S^{1}$-invariant polynomial of degree four is given by

$$
P_{c}=\sum_{j=0}^{4} c_{j} z_{1}^{j} z_{2}^{4-j},
$$

where $c=\left(c_{0}, \ldots, c_{4}\right) \in C^{5}$. The first term $A_{1}$ of an FES for $(C \oplus C, C)$ is the discriminant locus associated to homogeneous polynomials of degree four in two variables. Off the discriminant locus, $\boldsymbol{P}_{c}$ has four distinct roots corresponding to four distinct complex lines in $C \oplus C$. If we are given two sets of four distinct complex lines in $C \oplus C$, we cannot generally find a real linear endomorphism of $C \oplus C$ taking one set onto the other. $P_{c}: C \oplus C \rightarrow$ $C$ is $S^{1}$-transverse to $0 \in C$ if and only if $c \notin A_{1}$. Now if $c \notin A_{1}$ and $P_{c}^{-1}(0)$ is differentiably isotopic to $P_{d}^{-1}(0)$ (on some neighbourhood of zero) the derivative of the isotopy at zero maps $P_{c}^{-1}(0)$ onto $P_{d}^{-1}(0)$. By the above observation, this clearly cannot happen for all $d$ in some neighbourhood of $c$, however small, and so we do not have differential stability.

\section{Transversality in $G$-manifolds. II: The density theorem.}

THEOREM 7.1. Let $M$ and $N$ be $G$-manifolds and $W$ be $a$-invariant submanifold of $N$. Then any $h \in C_{G}^{\infty}(M, N)$ may be $C^{s}$-approximated by a map $f \in C_{G}^{\infty}(M, N)$ which is $G$-transversal to $W, 1 \leqslant s \leqslant \infty$.

THEOREM 7.2. Let $M$ and $N$ be $G$-manifolds and $W$ be a closed $G$-invariant submanifold of $N$. Then we may find an open dense subset $Z \subset C_{G}^{\infty}(M, N ; r)$, $r>d$, such that if $f \in Z$, there exists a neighbourhood $N$ of $f$ in $Z$ such that $g^{-1}(W)$ is equivariantly isotopic to $f^{-1}(W)$ for all $g \in N$. ( $d$ is the integer defined before the statement of Theorem 6.2.) 
Proofs of TheOREMS 7.1 AND 7.2. Let $K$ be a compact subset of $M_{i}$ (for notation see $\S 6$ ). Transversality theory implies that

$$
\left\{h \in C_{G}^{\infty}(M, N ; s): h \pitchfork_{G} W \text { on } K\right\}
$$

is an open dense subset of $C_{G}^{\infty}(M, N ; s), s>d$ (cf. the proof of Lemma $\mathrm{A}$ in $\S 6)$. Each $M_{i}$ may be written as a countable union of compact sets. Since there are only countably many orbit types for $M$, the set of $G$-transversal maps contains a countable intersection of open dense subsets of $C_{G}^{\infty}(M, N ; s)$. Now $C_{G}^{\infty}(M, N ; \infty)$ is a Baire space (same proof as is given in [7] for $\left.C^{\infty}(M, N ; \infty)\right)$ and so the set of $G$-transversal maps is dense in the $C^{\infty}$-topology. A fortiori, in the $C^{s}$-topology, $s \geqslant 1$. Theorem 7.2 follows from Theorem 7.1 and Theorem 6.2.

\section{REFERENCES}

1. R. Abraham and J. Robbin, Transversal mappings and flows, Benjamin, New York, 1967. MR 39 \#2181.

2. G. E. Bredon, Introduction to compact transformation groups, Academic Press, New York, 1972.

2a. E. Bierstone, General position of equivariant maps, Trans. Amer. Math. Soc. (to appear).

3. M. J. Field, Equivariant dynamical systems, Ph.D. Thesis, Warwick Univ., 1970.

4. __ Equivariant dynamical systems, Bull. Amer. Math. Soc. 76 (1970), 1314-1318. MR 43 \#3583.

5. __ Transversalité dans les G-variétés, C. R. Acad. Sci. Paris Sér. A-B 282 (1976).

6. Singularity theory and equivariant dynamical systems, (Proc. Internat. Conf. on Dynamical Systems in Mathematical Physics, Rennes), Asterisque (to appear).

7. M. Golubitsky and V. Guillemin, Stable mappings and their singularities, Springer-Verlag, New York, 1973. MR 49 \#6269.

8. S. Kojasiewicz, Ensembles semi-analytiques, I.H.E.S., Bures-Sur-Yvette, France, 1964.

9. J. N. Mather, Notes on topological stability, Harvard Univ., Cambridge, Mass., 1970 (preprint).

10. _ Stratifications and mappings, Dynamical Systems (Proc. Sympos., Univ. of Bahia, Salvador, 1971), Academic Press, New York, 1973, pp. 195-232.

11. R. Palais, Slices and equivariant embeddings, Seminar on Transformation Groups (editor, A. Borel), Ann. of Math. Studies, no. 46, Princeton Univ. Press, Princeton, N.J., 1968.

12. T. Petrie, G-transversality, Bull. Amer. Math. Soc. 81 (1975), 721-722.

13. V. Poenaru, Deploiment (uni-) versal des fonctions G-invariantes, Orsay notes, No. 105 74-22.

14. ___ Stabilité structurelle equivariante (première partie), Orsay notes, No. 126, 75-30.

15. B. Teissier, Introduction to equisingularity problems, Proc. Sympos. Pure Math., vol. 29, Amer. Math. Soc., Providence, R.I., 1975, pp. 593-632.

16. R. Thom, Ensembles et morphismes stratifiés, Bull. Amer. Math. Soc. 75 (1969), 240-284. M!R 39 \#970.

17. J. C. Tougeron, Idéaux de fonctions différentiables, Springer-Verlag, New York and Berlin, 1972.

18. A. N. Varčenko, Theorems on the topological equisingularity of families of algebraic varieties and families of polynomial mappings, Izv. Akad. Nauk SSSR Ser. Mat. 36 (1972), 957-1019 = Math. USSR Izv. 6 (1972), 949-1008. MR 49 \#2725. 
19. A. G. Wasserman, Equivariant differential topology, Topology 8 (1969), 127-150. MR 40 \#3563.

20. M. J. Field, Stratifications of equivariant varieties, Bull. Austral. Math. Soc. 16 (1977), 279-295.

Mathematics Institute, University of Warwick, Coventry CV4 7AL, England

Current address: Department of Mathematics, University of Sydney, Sydney, N.S.W. 2006, Australia 